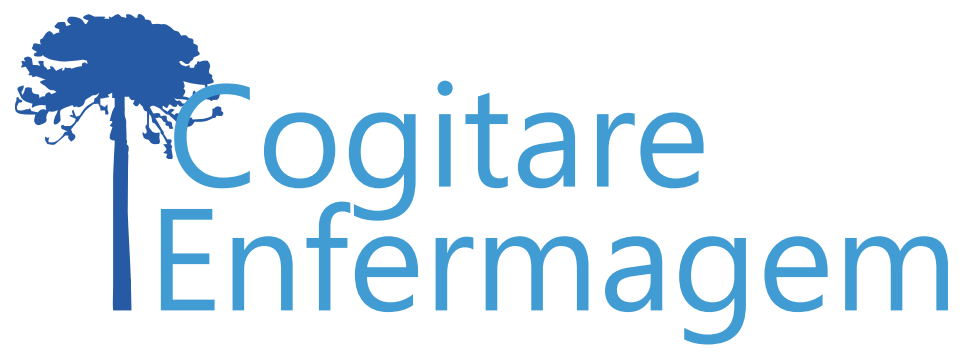

\title{
COMUNICAÇÃO E RELAÇÕES SOCIAIS ESTABELECIDAS ENTRE PAIS ADOECIDOS PELO CÂNCER E SEUS FILHOS
}

Michele Eugênio da Silva Vigarinho ${ }^{1}$, Mariângela Abate de Lara Soares ${ }^{2}$, Estela Ferreira da Silva ${ }^{3}$, Edvane Birelo Lopes De Domenico ${ }^{4}$

\section{RESUMO}

Objetivos: identificar os fatores intervenientes nas relações interpessoais e no processo de comunicação entre pais com câncer e seus filhos, relativos ao processo de adoecimento e descrever as estratégias favorecedoras.

Método: revisão integrativa, 25 artigos selecionados, entre 2007 e 2017 . Utilizou-se a estratégia de seleção: Patient, Intervention, Comparison e Outcomes para responder as questões norteadoras. Resultados: a funcionalidade da estrutura familiar, bem como as atitudes no adoecimento influenciam a comunicação dos pais com câncer e seus filhos. Na perspectiva dos profissionais, há falta de preparo para estes auxiliarem a família. Quando estratégias são empreendidas, os resultados geram impacto positivo.

Conclusão: a qualidade e efetividade da comunicação e das relações interpessoais são alcançadas com uma comunicação efetiva, direcionada e individualizada, amparada por equipe multidisciplinar preparada e articulada para auxiliar tanto os pais como seus filhos, além de estratégias presenciais, individuais e em grupo, bem como o uso de materiais educativos.

DESCRITORES: Neoplasias; Família; Criança; Relações familiares; Comunicação em Saúde.

COMO REFERENCIAR ESTE ARTIGO:

Vigarinho ME da S, Lara Soares MA de L, Silva EF da. Domenico EBL de. Comunicação e relações sociais estabelecidas entre pais adoecidos pelo câncer e seus filhos. Cogitare enferm. [Internet]. 2019 [acesso em "colocar data de acesso, dia, mês abreviado e ano"]; 24. Disponível em: http://dx.doi.org/10.5380/ce.v24i0.58645.

\section{(c) (1)}

Este obra está licenciado com uma Licença Creative Commons Atribuição 4.0 Internacional.

${ }^{1}$ Enfermeira. Especialista em Oncologia. Faculdade de Educação em Ciência da Saúde do Hospital Alemão Oswaldo Cruz. São Paulo, SP, Brasil. $\odot$

${ }^{2}$ Enfermeira. Doutora em Enfermagem. Docente da Universidade São Judas, Campus Unimonte e Universidade Católica de Santos. São Paulo, SP, Brasil. $\bigcirc$

${ }^{3}$ Enfermeira. Mestranda em Enfermagem. Enfermeira do A. C. Camargo Cancer Center. São Paulo, SP, Brasil. $\odot$

${ }^{4}$ Enfermeira. Doutora em Enfermagem. Docente de Enfermagem da Universidade Federal de São Paulo. São Paulo, SP,

Brasil. 


\title{
COMMUNICATION AND SOCIAL RELATIONS ESTABLISHED BETWEEN PARENTS WITH CANCER AND THEIR CHILDREN
}

\begin{abstract}
Objectives: To identify the intervening factors in interpersonal relations and in the process of communication between parents with cancer and their children, related to the disease process and describe the strategies that favor communication.

Method: Integrative review, with 25 articles selected between 2007 and 2017. The selection strategy: Patient, Intervention, Comparison and Outcomes was used to answer the guiding questions.

Results: The functionality of the family structure, as well as the attitudes taken during the disease process impact the communication of parents with cancer and their children. Health professionals claim they are not prepared to assist these families. When strategies are undertaken, the results generate positive impact.

Conclusion: The quality and effectiveness of communication and interpersonal relationships are achieved through effective and individualized communication, supported by a trained and well-coordinated multidisciplinary team that can assist both the parents and their children, in addition to face-to-face, individual and group strategies, as well as the use of educational materials.
\end{abstract}

DESCRIPTORS: Neoplasias; Family; Child; Family relationships; Health Communication.

\section{COMUNICACIÓN Y RELACIONES SOCIALES ESTABLECIDAS ENTRE PADRES CON CANCER Y SUS HIJOS}

\author{
RESUMEN: \\ Objetivos: identificar los factores que interfieren en las relaciones interpersonales y en el \\ proceso de comunicación entre padres con cáncer y sus hijos, en lo que se refiere al proceso \\ de enfermedad y describir las estrategias favorables. \\ Método: revisión integral, de 25 artículos seleccionados, entre 2007 y 2017. Se utilizó la \\ estrategia de selección: Patient, Intervention, Comparison y Outcomes para contestar a las \\ preguntas orientadoras. \\ Resultados: la funcionalidad de la estructura familiar, así como las actitudes en el proceso de \\ enfermedad influencian la comunicación de los padres con cáncer y sus hijos. En la perspectiva \\ de los profesionales, hace falta preparación para que estos ayuden a la familia. Cuando se \\ utilizan estrategias, los resultados generan impacto positivo. \\ Conclusión: se puede alcanzar la cualidad y efectividad de la comunicación y de las relaciones \\ interpersonales por medio de una comunicación efectiva, direccionada y individualizada, con \\ soporte del equipo multidisciplinar preparado y articulado para ayudar tanto a los padres \\ como a sus hijos, además de adoptarse estrategias presenciales, individuales y en grupo, así \\ como el uso de materiales educativos.
}

DESCRIPTORES: Neoplasias; Familia; Niños; Relaciones familiares; Comunicación en Salud. 
Segundo o Instituto Nacional do Câncer (INCA), a estimativa de casos novos de neoplasias malignas no Brasil entre os anos de 2016 e 2017, excetuando-se o câncer de pele não melanoma, foi de aproximadamente $600 \mathrm{~m}^{(1)}$. O crescimento dos casos é problema de saúde pública, não só por ser custoso para a sociedade sob diversos aspectos, mas porque acomete também crianças e adultos, estes, por vezes, em pleno estado de constituição familiar e produtividade profissional(1-2).

O câncer é uma doença estigmatizada socialmente. Há a crença no risco iminente de morte, e o receio de tratamentos agressivos e por vezes mutilantes, de longa duração, e que geram culpa e exclusão social(3). Além disso, muitas vezes o atraso faz com que - diagnóstico ocorra em estágios tardios da doença, nos quais a possibilidade de cura se inviabiliza - reforçando as crenças negativas. Assim, pacientes podem apresentar sentimentos diversos, como frustração, conflitos, inseguranças, crises de instabilidade e depressão(4).

O paciente com câncer não adoece sozinho. O primeiro impacto emocional acontece no diagnóstico ${ }^{(5-6)}$. Trata-se de um momento de crise e de incertezas, e o apoio da família é fundamental, pois são necessários ajustes e mudanças para incorporar os cuidados e o tratamento ${ }^{(3)}$. No caso do adulto jovem, várias situações sociais que requerem novas formas de condução ou adequação se somam, incluindo as relações familiares. A família tem sido foco de vários estudos em oncologia, o que denota a importância de mantê-la informada, capacitada para o gerenciamento dos cuidados, oferecendo apoio de natureza psíquica, física e material(7-8).

Nessa perspectiva, o cuidado integral inclui o olhar para a família, e as diferentes faixas etárias que a podem compor. A literatura científica evidencia que a comunicação entre pais adoecidos pelo câncer e seus filhos pode ser proativa ou problemática, gerando resultados imediatos e tardios nos sentimentos e comportamentos das crianças e adolescentes ${ }^{(9)}$.

A comunicação entre pais e filhos, no contexto do câncer, ainda é campo pouco explorado no que diz respeito aos fatores intervenientes dessa situação problema, bem como aos resultados nas relações interpessoais. Nessa perspectiva, teceram-se as perguntas do estudo: quais são as consequências nas relações interpessoais entre pais com câncer e seus filhos durante o processo de adoecimento? Quais são os principais fatores intervenientes para a qualidade e efetividade da comunicação e das relações interpessoais? Quais estratégias foram empreendidas para minimizar os problemas?

Assim, o estudo objetiva identificar os fatores intervenientes nas relações interpessoais e no processo de comunicação entre pais com câncer e seus filhos, relativos ao processo de adoecimento pelo câncer, e descrever as estratégias encontradas para o favorecimento da comunicação e da qualidade das relações interpessoais.

\section{MÉTODO}

Para a realização deste estudo, optou-se pela revisão integrativa da literatura, pensando que a síntese do conhecimento sobre o assunto possibilitaria a tomada de decisão do ponto de vista prático, e também revelaria as lacunas e necessidades de investimentos científicos ${ }^{(10)}$.

O método empregado foi constituído por seis etapas: identificação do tema; elaboração das hipóteses ou questões para a revisão integrativa; estabelecimento de critérios para inclusão e exclusão na literatura e seleção de amostra; apresentação das características dos estudos selecionados e análise crítica dos estudos incluídos; discussão dos resultados; apresentação da revisão(10). 
Para favorecer e qualificar a busca na literatura, as perguntas de estudo foram convertidas no acrônimo PICO, que significa Patient, Intervention, Comparison, Outcomes ${ }^{(11)}$. Assim, a "população" constituiu-se pelos pais com câncer e seus filhos, crianças/adolescentes. A "intervenção" relacionou-se com as descrições de estratégias (ou sua ausência) utilizadas para o favorecimento das relações interpessoais e da comunicação. A "comparação" baseou-se nos resultados gerados. O "resultado" ou desfecho vinculouse à qualidade das relações e ao alcance da comunicação efetiva.

A partir desta clareza intencional, procedeu-se às etapas subsequentes. A escolha dos descritores foi realizada a partir da definição e análise da pertinência em relação ao tema da investigação, utilizando-se os descritores em Ciências da Saúde (DeCS), isolados e combinados: neoplasia, família, criança, relações familiares e comunicação em saúde; nas bases de dados e ou bibliotecas eletrônicas Literatura Latino-americana e do Caribe em Ciências da Saúde (LILACS), Publiç Medline (PUbMed), Medical Literature Analysis and Retrieval System Online (MEDLINE), Índice Bibliográfico Espanhol de Ciências da Saúde (IBECS) e Scientific Electronic Library Online (ScIELO).

Os critérios de inclusão de artigos para este estudo foram: período de publicação entre 2007 a 2017, últimos 128 meses, em periódicos nacionais e internacionais (português e inglês); versar sobre pais com câncer de qualquer etiologia, em qualquer fase do processo de adoecimento, do diagnóstico ao seguimento, com filhos na faixa etária de 3 a 18 anos no período de adoecimento parental por câncer.

Não houve limitações em relação ao tipo de estudo, porém preocupou-se em classificar o nível de evidência, utilizando-se para tal o classificador disponibilizado pelo Instituto Joanna Briggs ${ }^{(12)}$. Inicialmente obteve-se os resumos dos artigos a partir dos descritores, e então procedeu-se à análise da adequação para responder às perguntas de estudo, considerando a opinião de 2 pesquisadores que realizaram a leitura de forma independente. O PICO foi utilizado para esta tarefa, favorecendo a inclusão ou a exclusão dos mesmos. A inclusão final foi decidida após a leitura de todos os artigos na íntegra e a seleção dos conteúdos que respondiam às questões de estudo.

\section{RESULTADOS}

Foram identificados 67 artigos que correspondiam à proposta inicial. Desses, 29 foram lidos na íntegra e, ao final, 25 artigos aplicaram-se ao desenho da revisão. O processo de seleção está descrito na Figura 1. 


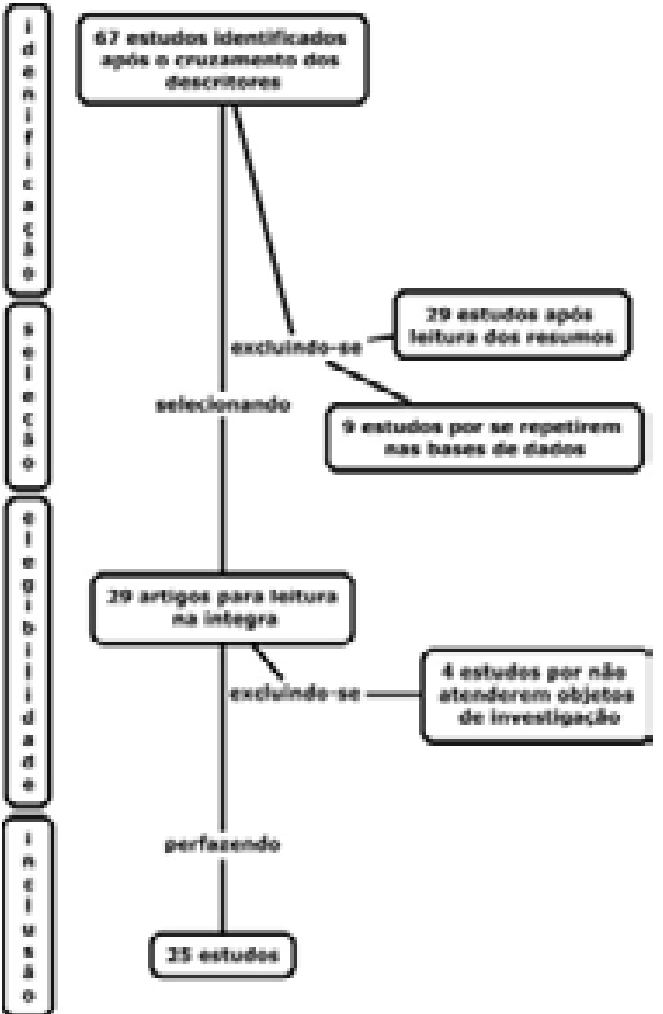

Figura 1 - Representação das etapas de elegibilidade dos artigos científicos. São Paulo, SP, Brasil, 2018

Do total, 20 artigos (80\%) foram publicados nos anos de 2010 a 2017, somente 20\% antes desse período. A maior parte está na língua inglesa $n=23(91,6 \%)$, tendo como método principal os estudos de natureza qualitativa. Os países de publicação dos estudos foram: Estados Unidos da América, Irã, Inglaterra, Brasil, Portugal, Noruega, Alemanha, Reino Unido, Porto Rico, Suécia, Austrália, Holanda. O Quadro 1 traz os manuscritos selecionados e os conteúdos extraídos de forma sumarizada e correspondente aos objetivos de estudo.

Quadro 1 - Síntese dos artigos selecionados de acordo com as perguntas de estudo. São Paulo, SP, Brasil, 2018 (continua)

\begin{tabular}{|c|c|c|}
\hline Artigos & Principais resultados sobre comunicação e & interação social: câncer parental e filhos \\
\hline $\begin{array}{l}\text { Referência/ } \\
\text { Ano/Nível } \\
\text { de Evidência }\end{array}$ & $\begin{array}{l}\text { Fatores intervenientes e/ou } \\
\text { consequências }\end{array}$ & Fatores e/ou estratégias favorecedoras \\
\hline $\begin{array}{l}\text { (13) } \\
2007 \\
\text { NE } 3\end{array}$ & $\begin{array}{l}\text {-Filhas de mães com câncer de mama } \\
\text { apresentavam mais sintomas depressivos } \\
\text { do que os filhos } \\
\text {-Mães e filhos com ansiedade, afetividade } \\
\text { negativa e depressão }\end{array}$ & $\begin{array}{l}\text {-Apoio dos amigos das mães e de } \\
\text { profissionais: menos sintomas depressivos } \\
\text { nas crianças } \\
\text {-Importância da família no ajuste e } \\
\text { adaptação individual }\end{array}$ \\
\hline $\begin{array}{l}(14) \\
2008 \\
\text { NE } 3\end{array}$ & $\begin{array}{l}\text {-Problemas: tratamento, morte, } \\
\text { responsabilidade da criação dos filhos } \\
\text {-A Qualidade de Vida apresentada pelos } \\
\text { pais foi refletiva no comportamento físico } \\
\text { e psicossocial dos filhos, dependendo da } \\
\text { idade da criança e da fonte de informações }\end{array}$ & $\begin{array}{l}\text {-Necessidade de investigar o efeito } \\
\text { de diferentes formas de câncer, seu } \\
\text { estágio, o prognóstico e tratamento na } \\
\text { funcionalidade dos membros da família, de } \\
\text { forma individual }\end{array}$ \\
\hline
\end{tabular}




\begin{tabular}{|c|c|c|}
\hline & $\begin{array}{l}\text {-Mães (mais que os pais) atentas aos } \\
\text { problemas gerados nas crianças } \\
\text {-Adolescentes: maior compreensão do } \\
\text { processo de adoecimento }\end{array}$ & \\
\hline $\begin{array}{l}(15) \\
2008 \\
\text { NE } 4\end{array}$ & $\begin{array}{l}\text {-As crianças sabiam o nome da doença } \\
\text { dos pais e percebiam o adulto sadio } \\
\text { sobrecarregado } \\
\text {-Envolvimento na hospitalização dos pais } \\
\text {-Falta de orientação para os pais nos } \\
\text { hospitais } \\
\text {-Comunicação emocional limitada }\end{array}$ & $\begin{array}{l}\text {-Estratégias de enfrentamento: "ajudar os } \\
\text { outros", identificar-se, distração, "manter } \\
\text { a doença em sua cabeça" e pensamentos } \\
\text { positivos } \\
\text {-Informação específica para as crianças }\end{array}$ \\
\hline $\begin{array}{l}(16) \\
2009 \\
\text { NE } 4\end{array}$ & $\begin{array}{l}\text {-Crianças: chateadas e tristes pela doença } \\
\text { da mãe, meninas preocupadas com a } \\
\text { própria saúde e com a da mãe. } \\
\text {-Expressaram angústia, estresse e } \\
\text { necessidade de apoio } \\
\text {-Pais/cuidadores: procurar manter a } \\
\text { normalidade diminuiu o impacto nas } \\
\text { crianças } \\
\text {-Câncer: pode gerar o fortalecimento das } \\
\text { relações familiares }\end{array}$ & $\begin{array}{l}\text {-Informação precisa } \\
\text {-Enfrentamento: mais tempo em família, } \\
\text { distração, normalidade e a presença dos } \\
\text { amigos } \\
\text {-Comunicação aberta: informar e conversar } \\
\text {-Explorar o impacto na vida das crianças e } \\
\text { suas estratégias de enfrentamento }\end{array}$ \\
\hline $\begin{array}{l}(17) \\
2009 \\
\text { NE } 4\end{array}$ & $\begin{array}{l}\text {-Filhos: transtorno do estresse pós- } \\
\text { traumático resultante da doença dos pais } \\
\text {-Efeitos adversos: sentimento de perda e } \\
\text { vazio, preocupações com a saúde pessoal, } \\
\text { piora da perspectiva da vida e negação } \\
\text { - Benefícios: maior apreciação da vida, } \\
\text { interesse em questões oncológicas }\end{array}$ & -Relações pessoais fortalecidas \\
\hline $\begin{array}{l}(18) \\
2010 \\
\text { NE } 4\end{array}$ & $\begin{array}{l}\text {-Diagnóstico e efeitos adversos: } \\
\text { insegurança, culpa, preocupação com o } \\
\text { bem-estar dos filhos, esforço, tristeza } \\
\text {-Mulheres: cuidado da criança e tarefas, } \\
\text { falta de apoio emocional do parceiro, } \\
\text { valorizado tempo em família } \\
\text {-Dificuldade na comunicação: falta de } \\
\text { confiança e sofrimento com a nova situação } \\
\text { de adoecimento, crença de que as crianças } \\
\text { não entendem, evitação de diálogo, } \\
\text { desesperança e falta de suporte pelos } \\
\text { profissionais }\end{array}$ & $\begin{array}{l}\text {-Rotina diária: proteção e conforto } \\
\text {-Familiares, amigos e redes sociais: } \\
\text { manter o funcionamento da casa e evitar } \\
\text { expectativas nas filhas } \\
\text {-Falar: aumenta a confiança e alivia aflições, } \\
\text { dizer a verdade e proteger a criança } \\
\text {-Equipe multiprofissional: estratégias para } \\
\text { os pais conversarem com seus filhos } \\
\text {-Adaptar as informações de acordo com a } \\
\text { idade e desenvolvimento da criança }\end{array}$ \\
\hline $\begin{array}{l}(19) \\
2010 \\
\text { NE } 4\end{array}$ & $\begin{array}{l}\text {-Mães e filhos: aflição, angústia, medo pelo } \\
\text { futuro, desconforto na autoimagem das } \\
\text { mulheres } \\
\text {-Crianças: agressividade, rebeldia, choros } \\
\text { sem causa, adesão a práticas religiosas, } \\
\text { brigas na escola e rendimento escolar } \\
\text { baixo. Sinais de ansiedade, depressão ou } \\
\text { estresse. Sentimento de exclusão por não } \\
\text { saberem o diagnóstico dos pais }\end{array}$ & $\begin{array}{l}\text { - Repensar as formas de atendimento e de } \\
\text { orientação as pacientes } \\
\text {-Atendimento integral em saúde: auxiliar as } \\
\text { mães a despertar os recursos necessários } \\
\text { para o enfrentamento da situação } \\
\text {-Relação aberta com os filhos }\end{array}$ \\
\hline $\begin{array}{l}\text { (20) } \\
2010 \\
\text { NE } 3\end{array}$ & $\begin{array}{l}\text { - } 16 \% \text { dos adolescentes com elevados } \\
\text { níveis de sintomas de resposta ao estresse } \\
\text { aos } 6 \text { meses após o diagnóstico do cancer } \\
\text { parental e } 14 \% \text { após } 1 \text { ano } \\
\text {-Filhas: maiores problemas } \\
\text {-Pais desconhecem o sofrimento dos filhos }\end{array}$ & $\begin{array}{l}\text { - Capacitar os pais para reconhecer os } \\
\text { problemas psicossociais em seus filhos } \\
\text {-Profissionais de Saúde em Oncologia } \\
\text { treinados para atuarem em relação ao } \\
\text { impacto do câncer parental sobre as } \\
\text { crianças e adolescentes }\end{array}$ \\
\hline
\end{tabular}




\begin{tabular}{|c|c|c|}
\hline $\begin{array}{l}(21) \\
2010 \\
\text { NE } 2\end{array}$ & $\begin{array}{l}\text {-Apoio social: escuta sensível, compreensão } \\
\text { e incentivo } \\
\text {-Importante: individualizar as formas de } \\
\text { apoio social para atender às necessidades } \\
\text { da criança e família, avaliar a idade e o } \\
\text { desenvolvimento da criança }\end{array}$ & $\begin{array}{l}\text {-Avaliar resolutividade e efetividade do } \\
\text { apoio social } \\
\text {-Estudos: tipos de apoio social durante } \\
\text { cada fase do câncer parental } \\
\text {-Preparo e educação de profissionais de } \\
\text { saúde sobre as várias formas de apoio } \\
\text { social }\end{array}$ \\
\hline $\begin{array}{l}\text { (22) } \\
2010 \\
\text { NE } 4\end{array}$ & $\begin{array}{l}\text {-Pais: preocupações sobre o futuro de seus } \\
\text { filhos; pressão sobre seus papéis somada } \\
\text { às demandas da doença } \\
\text {-Maior tensão com crianças jovens }\end{array}$ & $\begin{array}{l}\text {-Identificar necessidades psicossociais e } \\
\text { realizar encaminhamentos } \\
\text { - Obter referências de serviços de suporte } \\
\text { adequados }\end{array}$ \\
\hline $\begin{array}{l}(23) \\
2011 \\
\text { NE } 4\end{array}$ & $\begin{array}{l}\text {-Adolescentes: queixas somáticas, } \\
\text { problemas emocionais (críticos nos } \\
\text { primeiros } 4 \text { meses) principalmente em } \\
\text { meninas, medo da recidiva } \\
\text {-Contato restrito à equipe de oncologia: } \\
\text { crianças sentem-se ignoradas, despreparo } \\
\text { dos profissionais de saúde para ajudarem } \\
\text {-Depressão e aflição dos pais: problemas } \\
\text { psicossociais nos filhos }\end{array}$ & $\begin{array}{l}\text {-Redes de apoio para o paciente com } \\
\text { câncer: cônjuges, familiares, amigos e } \\
\text { profissionais da saúde } \\
\text {-Enfrentamento: rotina e atenção nas } \\
\text { respostas emocionais } \\
\text {-Ajustes: maior satisfação conjugal, tempo } \\
\text { de qualidade com os filhos, aprofundar as } \\
\text { relações e a comunicação } \\
\text {-Conscientização/ } \\
\text { treinamento da equipe: investigar, } \\
\text { acompanhar e auxiliar famílias } \\
\text {-Apoio profissional: disponibilizar tempo } \\
\text { e espaço adequado para a interação, } \\
\text { individualizar e construir vínculo com os } \\
\text { pais }\end{array}$ \\
\hline $\begin{array}{l}(24) \\
2011 \\
\text { NE } 4\end{array}$ & $\begin{array}{l}\text {-Pais: insatisfação, estresse, depressão, } \\
\text { baixa autoestima } \\
\text {-Crise no diagnóstico e tratamento } \\
\text {-Stress familiar: provocado pela } \\
\text { necessidade de comparecimento } \\
\text { frequente ao hospital, efeitos secundários } \\
\text { do tratamento, aumento dos encargos } \\
\text { financeiros, perda ou abalo do papel social } \\
\text {-Subestimar/desconhecer dificuldades dos } \\
\text { filhos para lidarem com o câncer } \\
\text {-Filhos dependentes: possibilidade de } \\
\text { apresentarem transtorno de stress pós- } \\
\text { traumático }\end{array}$ & $\begin{array}{l}\text {-Maior investigação acerca do tema e de } \\
\text { novos estudos com experiências familiares } \\
\text { positivas com o câncer }\end{array}$ \\
\hline $\begin{array}{l}(25) \\
2012 \\
\text { NE } 3\end{array}$ & $\begin{array}{l}\text {-Paternidade e Maternidade tardias elevam } \\
\text { o número de crianças e adolescentes que } \\
\text { experienciam a morte parental por câncer } \\
\text {-Diálogo e enfrentamento: dependentes } \\
\text { do nível de literacia em saúde e } \\
\text { do entendimento da condição de } \\
\text { adoecimento } \\
\text {-Crianças: temem a morte dos pais } \\
\text {-Não subestimar nas crianças e } \\
\text { adolescentes as consequências adversas e } \\
\text { capacidade de percepção da realidade }\end{array}$ & $\begin{array}{l}\text {-Assistência adequada: caracterizar } \\
\text { adequadamente os membros da família, } \\
\text { numericamente e qualitativamente } \\
\text {-Programas de apoio para promover/ } \\
\text { favorecer a comunicação nas famílias } \\
\text {-Conselho geral: comunicação aberta e } \\
\text { normalidade no funcionamento familiar }\end{array}$ \\
\hline $\begin{array}{l}(26) \\
2012 \\
\text { NE } 4\end{array}$ & $\begin{array}{l}\text {-Estratégia negativa ante o câncer parental: } \\
\text { negação } \\
\text {-Estratégias positivas: aceitação da doença, } \\
\text { acomodação do fator estressor, prática } \\
\text { religiosa/espiritual, busca por apoio } \\
\text { emocional }\end{array}$ & $\begin{array}{l}\text { - Identificação do problema de maior } \\
\text { impacto e adoção de estratégias para } \\
\text { minimizá-lo. } \\
\text {-Necessidade de intervenções psicológicas } \\
\text { adequadas para crianças de pais com } \\
\text { câncer, de acordo com a faixa etária. }\end{array}$ \\
\hline
\end{tabular}




\begin{tabular}{|c|c|c|}
\hline $\begin{array}{l}\text { (27) } \\
2013 \\
\text { NE } 4\end{array}$ & $\begin{array}{l}\text {-Crianças novas: foco na educação e apoio } \\
\text { emocional } \\
\text {-Barreiras no apoio centrado na família: } \\
\text { falta de conhecimento, preferência para } \\
\text { falar com um médico sobre os problemas } \\
\text { e subestimar o sofrimento dos filhos em } \\
\text { relação ao câncer }\end{array}$ & $\begin{array}{l}\text {-Acompanhamento psicossocial } \\
\text {-Educar os profissionais de saúde, } \\
\text { planejar cuidados de saúde, identificar } \\
\text { as famílias sob risco, informar os recém } \\
\text { diagnosticados sobre os serviços } \\
\text { oferecidos }\end{array}$ \\
\hline $\begin{array}{l}\text { (28) } \\
2013 \\
\text { NE } 3\end{array}$ & $\begin{array}{l}\text {-Adolescentes: pais subestimam seus } \\
\text { problemas e sentimentos } \\
\text {-Enfrentamento: associado à saúde mental } \\
\text { dos pais, inclusive } \\
\text {-Processo consciente de luto antecipado: } \\
\text { reduzir os problemas mentais nos pais e } \\
\text { filhos }\end{array}$ & $\begin{array}{l}\text {-Estratégias: aceitação, reestruturação } \\
\text { cognitiva e busca de apoio social } \\
\text {-Conversar e avaliar os filhos } \\
\text { individualmente } \\
\text {-Programas: espectro amplo de estratégias } \\
\text { baseadas em comunicação aberta }\end{array}$ \\
\hline $\begin{array}{l}\text { (29) } \\
2013 \\
\text { NE } 4\end{array}$ & $\begin{array}{l}\text {-Demanda por identificação das } \\
\text { necessidades não atendidas de jovens } \\
\text { acima de } 12 \text { anos para auxiliar na prestação } \\
\text { de serviços de assistência e suporte } \\
\text { direcionados na vigência do câncer } \\
\text { parental }\end{array}$ & $\begin{array}{l}\text { - criação e validação do instrumento } \\
\text { "Offspring Cancer Needs Instrument } \\
\text { (OCNI)", contendo } 47 \text { itens e } 7 \text { domínios: } \\
\text { information, family issues, practical } \\
\text { assistance, time out, feelings, support } \\
\text { (friends) and support (other young people) }\end{array}$ \\
\hline $\begin{array}{l}\text { (30) } \\
2014 \\
\text { NE } 3\end{array}$ & $\begin{array}{l}\text {-Funcionamento geral da família: } \\
\text { sintomático em crianças e seus pais } \\
\text {-Adolescentes de } 11-13 \text { anos com mais } \\
\text { sintomas do que os mais velhos } 14-21 \text { anos } \\
\text {-Crianças menores dependem mais do } \\
\text { apoio dos pais e são mais vulneráveis às } \\
\text { mudanças no funcionamento familiar }\end{array}$ & $\begin{array}{l}\text {-Estudos: fatores de risco e resiliência em } \\
\text { crianças } \\
\text {-Programas estruturados: comunicação } \\
\text { familiar, afetiva, envolvimento de membros } \\
\text { da família, resolução flexível de problemas, } \\
\text { apoio mútuo, e questões parentais na } \\
\text { prática clínica } \\
\text {-Comunicação aberta, afetividade e } \\
\text { funcionamento familiar flexível }\end{array}$ \\
\hline $\begin{array}{l}\text { (31) } \\
2014 \\
\text { NE } 4\end{array}$ & $\begin{array}{l}\text {-63\% dos pais observam angústia nos filhos } \\
\text {-Crianças: transtorno do estresse pós- } \\
\text { traumático, resiliência e maior apreço pela } \\
\text { vida (aumento da força pessoal) } \\
\text {-Mãe doente: baixa qualidade de vida } \\
\text {-Adolescentes: inseguros quanto à própria } \\
\text { vida } \\
\text {-Pais sem parceiros e com câncer: piora na } \\
\text { qualidade de vida das crianças }\end{array}$ & $\begin{array}{l}\text {-Pesquisa adicional: determinar a natureza } \\
\text { das necessidades não satisfeitas e obter } \\
\text { mais introspecção para que o apoio seja } \\
\text { adequado para sobreviventes, famílias e } \\
\text { crianças } \\
\text {-Instrumentos: investigar o bem-estar e } \\
\text { utilizar ferramentas para triagem }\end{array}$ \\
\hline $\begin{array}{l}\text { (32) } \\
2014 \\
\text { NE } 4\end{array}$ & $\begin{array}{l}\text {-Adolescentes: angústia, ansiedade e } \\
\text { depressão, menor autoestima } \\
\text {-Maiores impactos: doença avançada dos } \\
\text { pais, pobreza, abertura para comunicação, } \\
\text { idade (precoce versus final da adolescência) }\end{array}$ & $\begin{array}{l}\text {-Enfrentamento: distração para manter a } \\
\text { normalidade, falar sobre o câncer ou não } \\
\text { falar, raciocínio, ajudar o pai doente, busca } \\
\text { de significado e apoio social } \\
\text {-Perspectiva positiva, esperança } \\
\text {-Fornecer aos pais materiais (folhetos) } \\
\end{array}$ \\
\hline $\begin{array}{l}\text { (33) } \\
2016 \\
\text { NE } 4\end{array}$ & $\begin{array}{l}\text {-Adolescentes: ansiedade e depressão, } \\
\text { câncer como uma doença fatal, } \\
\text { sanguessuga ou um terremoto, declínio } \\
\text { no rendimento/frequência escolar, ter que } \\
\text { assumir as responsabilidades parentais } \\
\text {-Crianças: maior potencial para } \\
\text { compreender a doença sem estigmatizar o } \\
\text { câncer }\end{array}$ & $\begin{array}{l}\text {-Adolescentes: adaptação e ajuda aos } \\
\text { pais, aproximaram-se deles, revitalizaram } \\
\text { suas crenças espirituais, participaram de } \\
\text { programas de assistência social } \\
\text {-Enfrentamento pela religião } \\
\text {-Médicos: explorar as implicações da } \\
\text { doença, para o bem-estar dos adolescentes }\end{array}$ \\
\hline $\begin{array}{l}(34) \\
2016 \\
\text { NE } 4\end{array}$ & $\begin{array}{l}\text { - Crianças, geralmente, suspeitam de algo } \\
\text { errado e a falta de informação leva a } \\
\text { processos fantasiosos }\end{array}$ & $\begin{array}{l}\text {-Comunicação: lidar com a doença e } \\
\text { reduzir o sofrimento psicológico }\end{array}$ \\
\hline
\end{tabular}




\begin{tabular}{|c|c|c|}
\hline & $\begin{array}{l}\text {-Falta de comunicação: piora a saúde } \\
\text { mental dos pais e dos filhos; agrava os } \\
\text { problemas }\end{array}$ & $\begin{array}{l}\text {-Enfrentamento: distração, pensamento } \\
\text { positivo, compartilhamento de atividades } \\
\text { entre pais e filhos, fortalecimento de } \\
\text { relações e redes de apoio, manutenção da } \\
\text { normalidade, companheirismo e avaliar o } \\
\text { risco da parentificação (filhos assumindo } \\
\text { posição dos pais) }\end{array}$ \\
\hline $\begin{array}{l}(35) \\
2017 \\
\text { NE } 4\end{array}$ & $\begin{array}{l}\text {-Rompimento na rotina diária das crianças } \\
\text { e adolescentes, problemas de sono e } \\
\text { sintomas de transtorno do estresse pós- } \\
\text { traumático, aumento das responsabilidades } \\
\text { pessoais, aumento do absenteísmo escolar, } \\
\text { agressões, raiva, ansiedade, confusão, } \\
\text { tristeza, incerteza e medo }\end{array}$ & $\begin{array}{l}\text { - Rede de suporte de adolescentes: } \\
\text { amigos, conselheiros, professores, pais, } \\
\text { outros colegas em situações semelhantes } \\
\text {-Fatores protetores: } \\
\text { comunicação aberta, adaptabilidade, } \\
\text { expressividade, coesão, diminuição dos } \\
\text { conflitos } \\
\text {-Estratégias: distração, pensamento } \\
\text { ilusório, passar tempo com os pais e ajudar } \\
\text {-Ajuda apropriada para a idade, } \\
\text { identificação do impacto diagnóstico, } \\
\text { formular e } \\
\text { articular pensamentos e encontrar aspectos } \\
\text { positivos situacionais }\end{array}$ \\
\hline $\begin{array}{l}(36) \\
2017 \\
\text { NE } 4\end{array}$ & $\begin{array}{l}\text { - Pais com câncer avançado: níveis mais } \\
\text { altos de depressão e ansiedade quando } \\
\text { comparados com pacientes sem filhos } \\
\text { - Medo de perder os marcos futuros na } \\
\text { vida de seus filhos ou a capacidade de } \\
\text { moldar o desenvolvimento deles } \\
\text {-A identidade parental foi um dos motivos } \\
\text { para prosseguir o tratamento }\end{array}$ & $\begin{array}{l}\text {-Apoio dos pais: família e amigos para } \\
\text { manter suas funções } \\
\text {-Normalidade em casa } \\
\text {-Passar para os filhos valores, lições de vida } \\
\text { e ter memórias duradouras } \\
\text {-Profissionais de saúde: focar nas } \\
\text { preocupações dos pacientes, considerar o } \\
\text { papel parental em doenças que limitam a } \\
\text { vida e inclusive nas decisões de tratamento }\end{array}$ \\
\hline $\begin{array}{l}(37) \\
2017 \\
\text { NE } 4\end{array}$ & $\begin{array}{l}\text {-Crianças em idade escolar e adolescentes: } \\
\text { querem mais informações sobre o } \\
\text { prognóstico dos pais e a melhor forma de } \\
\text { ajudá-los } \\
\text {-Percebem a doença mesmo sem } \\
\text { comunicação. Temem piorar a situação, } \\
\text { desejam expressar seus sentimentos e } \\
\text { preocupações }\end{array}$ & $\begin{array}{l}\text {-Idade dos filhos } \\
\text {-Terapia familiar e aconselhamento } \\
\text { em grupos: informações para os pais } \\
\text { conversarem com os filhos } \\
\text {-Adolescentes se beneficiaram ao } \\
\text { conversarem com enfermeiras oncológicas } \\
\text {-Convivência com outras crianças que } \\
\text { enfrentaram o câncer parental é benéfica }\end{array}$ \\
\hline
\end{tabular}

A presente revisão de literatura incluiu 25 artigos científicos, publicados entre 2007 e 2017, sendo a língua inglesa a de preponderância (com exceção de 2 artigos em língua portuguesa). Em termos metodológicos, o tipo mais frequente de estudo foi o observacional $(n=11)$, seguido por revisões de literatura $(n=7)$ e analítico $(n=7)$, o que condiz com os resultados analisados que ainda estão na fase de investigação do problema, com poucas iniciativas propositivas de intervenções, dificultando a possibilidade de decisão sobre o que pode ser feito de forma mais eficaz.

Entretanto, os estudos encontrados foram fundamentais para a descrição das estratégias já empregadas para a detecção de sintomas biopsicossociais e para o favorecimento da comunicação e da qualidade das relações interpessoais. Dentre as ferramentas diagnósticas, a construção do instrumento de avaliação de necessidades de jovens, com idade superior a 12 anos, denominado instrumento "Offspring Cancer Needs 
Instrument (OCNI)"(29). O OCNI foi desenvolvido ante a percepção de demandas urgentes de aprimoramento da capacidade dos profissionais da saúde de identificarem com maior precisão os problemas que afligem jovens, como as interrupções na escolaridade, o aumento de carga de trabalho versus tempo para sair ou praticar esportes e tensões extras vivenciadas no ambiente domiciliar ${ }^{(29)}$.

Com relação ao processo de comunicação entre pais com câncer e seus filhos, as investigações científicas sinalizaram que, normalmente, este ocorre de forma não planejada pelos pais e não assistida pela equipe de profissionais envolvidos no cuidado do adulto e da família. Representações sociais concernentes ao papel dos pais de proteger as crianças de notícias ruins, conflitos e doenças são citadas em várias publicações selecionadas ${ }^{(13-15,18-20)}$, porém essas atitudes algumas vezes geraram certos comportamentos negativos nas crianças, como fantasiarem negativamente a morte dos pais ou se sentirem excluídas da família ${ }^{(22-25,34)}$.

Para melhor compreensão do contexto das repercussões do câncer parental, além do impacto do diagnóstico que, por si, mobiliza emoções que remetem a condições de dor, sofrimento e morte em grande parte da população(38), ainda há a necessidade de enfrentamento dos efeitos secundários físicos do tratamento, rotinas de consultas, idas ao hospital, preparo de exames, aumento dos encargos financeiros ${ }^{(24)}$, entre outras condições que podem alterar a identidade familiar e os papéis já destinados a cada membro, pois existem alterações consideráveis na rotina das pessoas e na dinâmica familiar.

Portanto, o paciente e os familiares podem ficar suscetíveis ao aumento do stress, depressão, ansiedade e externalização dos sentimentos, por vezes de forma intempestiva. Considerando faixas etárias, as crianças menores, que são mais dependentes do apoio dos pais, são mais vulneráveis a essas mudanças e apresentam níveis mais elevados de transtornos do estresse pós-traumático ${ }^{(14,31,35)}$. Somando-se a isso, alterações de autoimagem como queda do cabelo e mastectomia radical atingiram não somente as mães, mas também suas filhas, principalmente, pela dificuldade em aceitar as novas condições ${ }^{(13,19,23)}$.

Ainda traçando o panorama na estrutura familiar, alguns artigos abordam o fato de que quando a doença afeta a mãe, traz maiores dificuldades aos filhos do que quando o pai é o doente. Isso se explica por a mãe, na maioria das vezes, ser a cuidadora central, estar mais próxima dos seus filhos e ser a mantenedora da rotina domiciliar. Entretanto, as consequências são piores para as crianças quando o pai recebe o diagnóstico, e uma das explicações é o pior prognóstico dos cânceres nos homens ${ }^{(39)}$, em decorrência, possivelmente, dos diagnósticos tardios resultando em doença avançad'a e metastática ${ }^{\left({ }^{29}\right)^{\prime}}$.

Os pais com câncer também apresentaram maiores dificuldades em identificar problemas em seus filhos do que as mães e até mesmo os professores, por terem menos contato com os filhos, estarem mais focados nas companheiras e na sua tendência de serem autoorientados ${ }^{(14)}$. Contudo, algumas mães com câncer relataram que a falta de apoio do parceiro/ pai ou a ausência de um, dificultou o relacionamento familiar. Apesar dessas particularidades, estudo(22) demonstrou que para o pai ou mãe com câncer há a angústia relacionada com o futuro dos filhos, na perspectiva de estarem amedrontados com a possibilidade de não estarem vivos para acompanharem o crescimento deles, bem como com relação a necessidade de terem que escolher alguém para transferir a responsabilidade.

Todos esses conflitos influenciam o modo e a forma como as crianças e adolescentes adaptam-se à doença dos pais. Ao vivenciar o câncer parental, eles podem apresentar problemas físicos e emocionais, como depressão, ansiedade, agressividade, rebeldia, choros sem causa aparente, adesão a questões religiosas incomuns à família, brigas na escola, declínio no rendimento escolar e na frequência das aulas. Alguns artigos focaram nos adolescentes, já que esta fase do desenvolvimento humano gera maiores habilidades cognitivas abstratas e exigências psicobiológicas conflitantes. Por isso, podem apresentar queixas somáticas, como fadiga, dor abdominal, tonturas, problemas com sono e alimentação. Além disso, podem sentir-se sobrecarregados com papéis e responsabilidades, sofrer com luto antecipado e ter interrupções em suas vidas diárias. Para tentar enfrentar 
a situação, pode haver negação, como forma de liberar o estresse pelo diagnóstico, ou ainda aceitação da doença e acomodação dos fatores estressores.

Inserida em todo esse contexto familiar, está a equipe de saúde responsável pelo cuidado do adulto com câncer. Além dos pais sentirem dificuldade de tratar o assunto com a equipe médica ou multidisciplinar e não receberem orientações em relação à comunicação com seus filhos, as crianças também se sentem ignoradas e com medo de compartilharem seus sentimentos sobre a doença parental, muitas vezes não tendo acesso aos profissionais envolvidos com o cuidado da família(15). Para a equipe, a falta de conhecimento adequado e direcionado ao público infantil/adolescente e a escassez de serviços psicossociais dificultam o diálogo com os pais e a adoção de estratégias na condução da relação com seus filhos ${ }^{(15)}$.

Uma das investigações acerca do tema trouxe resultados interessantes com relação ao processo de adoecimento pelo câncer, como o fortalecimento das relações familiares, o aumento do tempo destinado à família e o apoio às crianças/adolescentes para que essa fase gerasse experiências positivas ${ }^{(17)}$.

O segundo objetivo desta revisão era identificar fatores e estratégias já empregadas no favorecimento da comunicação e da qualidade das relações interpessoais. Houve um consenso entre os estudos sobre a necessidade de se considerar o estágio de desenvolvimento da criança e seus sentimentos para que a intervenção fosse efetiva e direcionada. Além disso, deve-se focar na família, na comunicação eficaz entre os membros (ajudando a manter a normalidade diária) e na busca ativa por famílias que enfrentam o câncer parental, para um apoio social adequado.

Algumas famílias também nutriram sua rede de apoio(40) para enfrentar o processo, com amigos, cônjuges, familiares e profissionais da saúde. Alguns estudos comprovaram a efetividade do apoio psicossocial que famílias de pais com câncer vivenciavam. Em um deles, $82 \%$ dos pacientes acham útil o acompanhamento e $73 \%$ dos sobreviventes que tinham crianças pequenas gostariam de apoio psicossocial para enfrentar o processo de adoecimento. O apoio psicossocial é um conjunto de estratégias sociais e psicológicas que complementam o tratamento farmacológico e o gerenciamento de sintomas, para melhorar o funcionamento social e pessoal, a qualidade de vida individual e familiar e a integração na comunidade ${ }^{(40)}$.

Os materiais educativos, que incluem folhetos ${ }^{(32)}$, destacam-se na orientação de crianças/adolescentes que enfrentam o câncer parental, por constituírem-se ferramentas que auxiliam os pais e familiares na comunicação efetiva acerca do processo de adoecimento. É necessária uma correspondência entre os interesses e necessidades dos leitores, a adequação da linguagem e das ilustrações, informações confiáveis e um vocabulário claro e objetivo, para a efetividade dos instrumentos. Além disso, a construção desse material deve ser feita com uma abordagem participativa, comunicativa e coletiva ${ }^{(41)}$.

Além de estratégias apontadas como favorecedoras, estudos presentes na revisão indicaram a necessidade de maiores evidências científicas, primando-se por pesquisas que considerem as abordagens comportamentais, estruturais e relacionados a diferentes formas e estágios da doença ${ }^{(14)}$. Também, novos desenhos observacionais e experimentais que investiguem o processo de enfrentamento do câncer parental, abordando os fatores de risco, o apoio psicossocial e a comunicação entre pais e filhos nas várias fases da doença e o processo de adoecimento do pai.

\section{CONCLUSÃO}

Esta revisão integrativa evidenciou que há consequências, predominantemente negativas, para a comunicação e as relações interpessoais entre pais com câncer e seus filhos, quando o processo se desenvolve de maneira a desconsiderar a necessidade da criança e ou adolescente de obterem informações e apoio de forma planejada, dialógica 
e amparada por pais, familiares e profissionais sensíveis a esta necessidade. Identificouse que os filhos podem apresentar problemas psicológicos, físicos e comportamentais a depender da forma de condução do processo de adoecimento parental. De acordo com as evidências, ignorar e tentar preservar os filhos, subtraindo-lhes informações e emoções, são atitudes que geram ansiedade, medo e angústia.

A qualidade e efetividade da comunicação e das relações interpessoais podem ser alcançadas desde o início da doença (fase diagnóstica ou terapêutica) de maneira direcionada e individualizada, se amparada por equipe interdisciplinar preparada e articulada para auxiliar pais e filhos. Estratégias presenciais, individuais e em grupo, bem como materiais educativos, podem favorecer essa dinâmica.

Pôde-se concluir que há lacunas na construção do conhecimento tanto na esfera do entendimento científico, como em relação às intervenções que previnam dificuldades na comunicação e nas relações entre pais e filhos durante o processo de adoecimento. Entretanto, a literatura analisada trouxe contribuições importantes para que o problema seja considerado e que ações sejam implementadas para a qualificação da assistência em oncologia.

Contribuições para a prática: os resultados confirmam a importância do cuidado ser estendido à família, em particular nas situações nas quais há crianças/adolescentes. Esse cuidado ampliado requer atenção multidisciplinar, capaz de capacitar o pai e/ou mãe para lidar satisfatoriamente com a comunicação e relacionamento com os filhos no processo de adoecimento pelo câncer.

\section{REFERÊNCIAS}

1. Instituto Nacional de Câncer José Alencar Gomes da Silva (INCA). Estimativa 2018: incidência de câncer no Brasil. Rio de Janeiro: INCA; 2018.

2. Stewart B, Wild CP. World Cancer Report 2014. International Agency for Research on Cancer. [Internet]. 2014 [acesso em 18 set 2016]. Disponível em: http://www.thehealthwell.info/node/725845.

3. Ulysses CSC. A necessária atenção à família do paciente oncológico. Rev. Bras. Cancerol. [Internet]. 2008 [acesso em 18 set 2016]; 54(1). Disponível em: http://www1.inca.gov.br/rbc/n 54/v01/pdf/ revisao 7 pag 97a102.pdf.

4. Bottino SMB, Fráguas R, Gattaz WF. Depressão e câncer. Rev. Psiquiatr. Clin. [Internet]. 2009 [acesso em 17 fev 2019]; 36(Suppl 3). Disponível em: http://dx.doi.org/10.1590/S0101-60832009000900007.

5. Martins CBS, Silva Filho N, Pires MLN. Estratégias de Coping e o impacto sofrido pela família quando um dos seus está em tratamento contra o câncer. Mudanças, Psicol. Saúde [Internet]. 2011 [acesso em 18 set 2016]; 19(1/2). Disponível em: http://dx.doi.org/10.15603/2176-1019/mud.v19n1-2p11-18.

6. Ferrazza A, Muniz RM, Pinto BK, Viegas AC, Matos MR. A sobrevivência ao câncer na perspectiva da família. Rev. Enferm. UFPE [Internet]. 2016 [acesso em set 2016]; 10(3). Disponível em: https://periodicos. ufpe.br/revistas/revistaenfermagem/article/viewFile/11054/12469.

7. CancerCare. Helping Children Understand cancer: talking about to your kids about your diagnosis. [Internet]. 2018 [acesso em 21 fev 2019]. Disponível em: https://media.cancercare.org/publications/ original/49-fs children eng.pdf.

8. Melo MCB, Barros EN, Campello MCVA, Ferreira LQL, Rocha LLC, Silva CIMG, eat al. O funcionamento familiar do paciente com câncer. Psicologia em Revista [Internet]. 2012 [acesso em 20 set 2016]; 18(1).

Disponivel em: https://doi.org/10.5752/P.1678-9563.2012v18n1p73.

9. Wakiuchi J, Ribeiro AL, Benedetti GMS, Merino MFGL, Marcon SS, Sales CA. Sentimentos dos filhos ao vivenciarem o adoecimento dos pais. Rev. Eletr. Enf. [Internet]. 2016 [acesso em 23 set 2017]; (18). 
10. Ganong LH. Integrative reviews of nursing research. Res. nurs. Health [Internet]. 1987 [acesso em 10 de nov de 2016]; 10(1). Disponível em: https://doi.org/10.1002/nur.4770100103.

11. Santos CMC, Pimenta CAM, Nobre MRC. The PICO strategy for the research question construction and evidence search. Rev. Latino-Am. Enfermagem [Internet]. 2007 [acesso em 20 set 2016]; 15(3). Disponível em: http://dx.doi.org/10.1590/S0104-11692007000300023.

12. The Joanna Briggs Institute. The JBI Approach. Grades of recommendation. Levels of Evidence [Internet]. Adelaide; 2014 [acesso em 11 nov 2016] Disponível: http://joannabriggs.org/jbi-approach. html\#tabbed-nav=Levels-of-Evidence.

13. Brown RT, Fuemmeler B, Anderson D, Jamieson S, Simonian S, Hall RK, et al. Adjustment of Children and Their Mothers with Breast Cancer. J Pediatr Psychol. [Internet]. 2007 [acesso em 10 out 2016]; 32(3). Disponível em: https://doi.org/10.1093/jpepsy/jsl015.

14. Gazendam-Donofrio SM, Hoekstra HJ, Van der Graaf WTA, Pras E, Visser A, Huizinga GA, HoekstraWeebers JEHM. Quality of life of parents with children living at home: when one parent has cancer. Support care cancer [Internet]. 2008 [acesso em 11 out 2016]; 16(2). Disponível em: https://dx.doi. org/10.1007/s00520-007-0299-7.

15. Thastum M, Johansen MB, Gubba L, Olesen LB, Romer G. Coping, Social Relations, and Communication: A Qualitative Exploratory Study of Children of Parents with Cancer. Clin Child Psychol Psychiatry [Internet]. 2008 [acesso em 9 nov 2016]; 13(1). Disponível em: https://doi. org/10.1177/1359104507086345.

16. Kennedy VL, Lloyd-Williams M. How children cope when a parent has advanced câncer. Psicho-oncol. [Internet]. 2009 [acesso em 10 nov 2016]; 18(8). Disponível em: https://doi.org/10.1002/pon.1455.

17. Wong ML, Cavanaugh CE, MacLeamy JB, Sojourner-Nelson A, Koopman C. Posttraumatic Growth and Adverse Long-Term Effects of Parental Cancer in Children. Fam Syst Health. [Internet]. 2009 [acesso em 10 nov 2016]; 27(1). Disponível em: http://dx.doi.org/10.1037/a0014771.

18. Semple CJ, McCance T. Parents' Experience of Cancer Who Have Young Children. Cancer Nurs. [Internet]. 2010 [acesso em 10 nov 2016]; 33(2). Disponível em: https://doi.org/10.1097/ NCC.0b013e3181c024bb.

19. de Castro EK, Job C. Câncer na Mãe e o Impacto Psicológico no Comportamento de seus Filhos Pequenos. Gerais, Rev. Interinst. Psicol. [Internet]. 2010 [acesso em 20 set 2016]; 3(2). Disponível em: http://www.fafich.ufmg.br/gerais/index.php/gerais/article/view/102.

20. Huizinga GA, Visser A, Van der Graaf WTA, Hoekstra HJ, Gazendam-Donofrio SM, Hoekstra-Weebers JEHN. Stress response symptoms in adolescents during the first year after a parent's cancer diagnosis. Support. care cancer [Internet] 2010 [acesso em 11 nov 2016]; 18(11). Disponível em: https://doi. org/10.1007/s00520-009-0764-6.

21. Wong M, Ratner J, Gladstone KA, Davtyan A, Koopman C. Children's Perceived Social Support After a Parent Is Diagnosed with Cancer. J Clin Psychol Med Settings [Internet]. 2010 [acesso em 11 nov 2016]; 17(2). Disponível em: https://doi.org/10.1007/s10880-010-9187-2.

22. Weaver KE, Rowland JH, Alfano CM, McNeel TS. Parental Cancer and the Family. Cancer [Internet]. 2010 [acesso em 11 nov 2016]; 116(18). Disponível em: https://doi.org/10.1002/cncr.25368.

23. Huizinga GA, Visser A, Zelders-Steyn YE, Teule JA, Sijmen A. Reijneveld SA, Roodbol PF. Psychological impact of having a parent with câncer. Eur J. Cancer. [Internet]. 2011 [acesso em acesso em 11 nov 2016]; 47(Suppl 3). Disponível em: https://doi.org/10.1016/S0959-8049(11)70170-8.

24. Teixeira RJ, Pereira MG. Impacto do Câncer Parental no Desenvolvimento Psicológico dos Filhos: Uma Revisão da Literatura. Psicol. Reflex. Crít. [Internet]. 2011 [acesso em 25 set 2016]; 24(3). Disponível em: http://dx.doi.org/10.1590/S0102-79722011000300012. 
25. Syse A, Aas GB, Loge JH. Children and young adults with parents with cancer: a population-based study. Clin. Epidemiol. [Internet]. 2012 [acesso em 11 nov 2016]; 4(1). Disponível em: https://doi. org/10.2147/CLEP.S28984.

26. Costas-Muñiz R. Hispanic adolescents coping with parental câncer. Support. care cancer [Internet]. 2012 [acesso em 11 nov 2016]; 20(2). Disponível em: https://doi.org/10.1007/s00520-011-1283-9.

27. Ernst JC, Beierlein V, Romer G, Möller B, Koch U, Bergelt C. Use and Need for Psychosocial Support in Cancer Patients. Cancer [Internet]. 2013 [acesso em 11 nov 2016]; 119(12). Disponível em: https://doi. org/10.1002/cncr.28021.

28. Krattenmacher T, Kühne F, Führer D, Beierlein V, Brähler E, Resch F, et al. Coping skills and mental health status in adolescents when a parent has cancer: A multicenter and multi-perspective study. J. psychosom. res. [Internet]. 2013 [acesso em 11 nov 2016]; 74(3). Disponível em: https://doi.org/10.1016/j. jpsychores.2012.10.003.

29. Patterson P, McDonald FEJ, Butow P, White KJ, Costa DSJ, Pearce A, et al. Psychometric evaluation of the Offspring Cancer Needs Instrument (OCNI): an instrument to assess the psychosocial unmet needs of young people who have a parent with cancer. Support. care cancer [Internet]. 2013 [acesso em 11 nov 2016]; 21(7). Disponível em: https://doi.org/10.1007/s00520-013-1749-z.

30. Möller B, Barkmann C, Krattenmacher T, Kühne F, Bergelt C, Beierlein V, et al. Children of Cancer Patients: Prevalence and predictors of emotional and behavioral problems. Cancer [Internet]. 2014 [acesso em 11 nov 2016]; 120(15). Disponível em: https://doi.org/10.1002/cncr.28644.

31. Bultmann JC, Beierlein V, Romer G, Moller B, Koch U, Bergel C. Parental cancer: Health-related quality of life and current psychosocial support needs of cancer survivors and their children. Int. J. Cancer [Internet]. 2014 [acesso em acesso em 11 nov 2016]; 135(11). Disponível em: https://doi.org/10.1002/ ijc.28905.

32. Phillips F. Adolescents living with a parent with advanced cancer: a review of the literature. Psychooncol. [Internet]. 2014 [acesso em 12 nov 2016]; 23(12). Disponível em: https://doi.org/10.1002/pon.3570.

33. Azarbarzin M, Malekian A, Taleghani F. Adolescents' Experiences When Living With a Parent With Cancer: A Qualitative Study. Iran Red Crescent Med J. [Internet]. 2016 [acesso em 12 nov 2016]; 18(1). Disponível em: https://doi.org/10.5812/ircmj.26410.

34. Morris JN, Martini A, Preen D. The well-being of children impacted by a parent with cancer: an integrative review. Support. Care Cancer [Internet]. 2016 [acesso em 12 nov 2016]; 24(7). Disponível em: https://doi.org/10.1007/s00520-016-3214-2.

35. Shah BY, Armaly J, Swieter E. Impact of Parental Cancer on Children. Anticancer Res. [Internet]. 2017 [acesso em 12 nov 2016]; 37(8). Disponível em: https://doi.org/10.21873/anticanres.11787.

36. Park EM, Check DK, Song MK, Reeder-Hayes KE, Hanson LC, Yopp JM, et al. Parenting while living with advanced cancer: A qualitative study. Palliative Medicine [Internet]. 2017 [acesso em 13 nov 2016]; 31(3). Disponível em https://doi.org/10.1177/0269216316661686.

37. Ellis SJ, Wakefield CE, Antill G, Burns M, Patterson P. Supporting children facing a parent's cancer diagnosis: a systematic review of children's psychosocial needs and existing interventions. Eur J Cancer Care [Internet]. 2017 [acesso em 13 nov 2016]; 26(1). Disponível em: https://doi.org/10.1111/ecc.12432.

38. de Oliveira WT, Benedetti GMS, Marchi JA, Cassarotti MS, Wakiuchi J, Sales CA. Eventos intensificadores e redutores do estresse em famílias de pacientes com câncer: revisão integrativa. Rev. Min. Enferm. [Internet]. 2013 [acesso em 14 nov 2016]; 17(3). Disponível em: http://www.dx.doi. org/10.5935/1415-2762.20130052.

39. Quaife, S. L., Winstanley, K., Robb, K. A., Simon, A. E., Ramirez, A. J., Forbes, L. J., et al. Socioeconomic inequalities in attitudes towards cancer: an international cancer benchmarking partnership study. Eur J Cancer Prev. [Internet]. 2015 [acesso em 14 out 2017]; 24(3). Disponível em: https://doi. org/10.1097/CEJ.0000000000000140. 
40. da Silva TFC. Avaliando a fidelidade de intervenções psicossociais: uma revisão sistemática da literatura. J. Bras. Psiquiatr. [Internet]. 2014 [acesso em 14 nov 2016]; 63(3). Disponível em: http://dx.doi. org/10.1590/0047-2085000000034.

41. Reberte LM, Hoga LAK, Gomes ALZ. Process of construction of an educational booklet for health promotion of pregnant women. Rev. Latino-Am. Enfermagem [Internet]. 2012 [acesso em 14 nov 2016]; 20(1). Disponível em: http://dx.doi.org/10.1590/S0104-11692012000100014.

Recebido: 29/03/2018

Finalizado: 19/03/2019

Autor Correspondente:

Michele Eugênio da Silva Vigarinho

Universidade Federal de São Paulo

R. Napoleão de Barros, 754 - 04024-002 - São Paulo, SP, Brasil

E-mail: Michele_2020@hotmail.com

Contribuição dos autores:

Contribuições substanciais para a concepção ou desenho do estudo; ou a aquisição, análise ou interpretação de dados do estudo - MESV, MALS, EFS

Elaboração e revisão crítica do conteúdo intelectual do estudo - MALS, EBLD

Aprovação da versão final do estudo a ser publicado - EBLD

Responsável por todos os aspectos do estudo, assegurando as questões de precisão ou integridade de qualquer parte do estudo - MESV, EBLD 